\title{
Complications of Convex-Probe Endobronchial Ultrasound-Guided Transbronchial Needle Aspiration: A Multi-Center Retrospective Study
}

\author{
Benan Çağlayan MD, Aydın Yılmaz MD, Semra Bilaçeroğlu MD, Sevda Şener Cömert MD, \\ Nilgün Y Demirci MD, and Banu Salepçi MD
}

\begin{abstract}
BACKGROUND: Infrequent serious complications of convex-probe endobronchial ultrasoundguided transbronchial needle aspiration (EBUS-TBNA) have been reported. The aim of this study was to assess serious complications related to convex-probe EBUS-TBNA and to determine the complication rate in a large group of subjects. METHODS: In this retrospective study, a 15-item questionnaire on features of cases with EBUS-TBNA complications was sent to experienced bronchoscopists performing convex-probe EBUS-TBNA at 3 pulmonary centers. The medical records were then reviewed by these bronchoscopists to complete the questionnaire. Hemorrhage responsive to topical treatment, temporary laryngospasm/bronchospasm, transient oxygen desaturation, and fever lasting $<24 \mathrm{~h}$ were excluded. Only complications requiring further treatment/intervention were considered serious. The rate of serious complications was calculated from the obtained data. RESULTS: In a total of 3,123 cases within a 5-y period, EBUS-TBNA was performed for staging lung cancer in $15.8 \%$, diagnosis in $67.5 \%$, and diagnosis and staging in $16.3 \%$. Of the $3,123,11.6 \%$ had parenchymal lesions adjacent to major airways. EBUS-TBNA was performed 11,753 times (3.76/case) at 6,115 lymph node stations and lesions (1.92/station or lesion). Five serious complications were recorded $(0.16 \%)$ : fever lasting $>24 \mathrm{~h}$, infection of bronchogenic cyst, mediastinal abscess, pericarditis, and pneumomediastinitis with empyema, each in one case. Four complications occurred in cases diagnosed with benign disease by EBUS-TBNA. All complications were treated with broad-spectrum antibiotics. Four subjects were hospitalized for $21.7 \pm 20.7$ d. CONCLUSIONS: Convex-probe EBUS-TBNA is a safe method in general. However, serious complications, including infections, can be encountered rarely. All precautions should be taken for complications before and during the procedure. Key words: endobronchial ultrasonography; transbronchial needle aspiration; complications. [Respir Care 2016;61(2):243-248. (C) 2016 Daedalus Enterprises]
\end{abstract}

\section{Introduction}

Convex-probe endobronchial ultrasound-guided transbronchial needle aspiration (EBUS-TBNA) is a minimally invasive method for investigating the mediastinum, for

Drs Çağlayan, Cömert, and Salepçi are affiliated with the Department of Pulmonary Diseases, Dr. Lütfi Kırdar Kartal Training and Research Hospital, Istanbul, Turkey. Drs Yilmaz and Demirci are affiliated with the Atatürk Chest Diseases and Thoracic Surgery Education and Research Hospital, Interventional Pulmonology, Ankara, Turkey. Dr Bilaçeroğlu is affiliated with the Department of Pulmonary Diseases, Izmir Dr. Suat Seren Training and Research Hospital for Thoracic Medicine and Surgery, Izmir, Turkey. staging of non-small-cell lung cancer, and for diagnosing mediastinal lesions accessible via the major airway. It is

\footnotetext{
Dr Çağlayan presented a version of this paper at the European Respiratory Society International Congress 2014, held September 6-10, 2014, in Munich, Germany.

The authors have disclosed no conflicts of interest.

Correspondence: Sevda Şener Cömert MD, Pembe Köşk sok. Emek apt. No:16 D:14 Merdivenköy Kadıköy-İstanbul 35732, Turkey. E-mail: sevdasener2@yahoo.com.
}

DOI: $10.4187 /$ respcare. 03838 
increasingly used because of its safety, simplicity, high diagnostic yield, and ability to diagnose both benign and malignant conditions. The cumulative sensitivity and specificity of EBUS-TBNA in the lymph node staging of lung cancer is $88-93 \%$ and $100 \%$, respectively. ${ }^{1}$

It is a noninvasive technique with minor complications of agitation, cough, and bleeding at the puncture site. ${ }^{2,3}$ Varela-Lema et $\mathrm{al}^{2}$ published a systematic review and noted no important complications related to $>1,500$ EBUSTBNA procedures. A meta-analysis on EBUS-TBNA reported a complication rate of 2 in 1,299 cases $(0.15 \%)$, with only 1 case showing pneumothorax requiring drainage as a major complication. ${ }^{4}$

EBUS-TBNA-related complications do occur, although the complication rate is low. Mediastinal abscess, empyema, mediastinal emphysema, pericarditis, sepsis, needle breakage, intramural hematoma of the pulmonary artery, hemopneumomediastinum, and lung abscess are major complications that have been reported in recent years, mostly as case reports., ${ }^{2,5-9}$ Systematic reviews report only 1 incidence of morbidity in a subject with pneumothorax after the procedure $\left(0.07 \%\right.$ morbidity) ${ }^{4,10} \mathrm{~A}$ few deaths following EBUS-TBNA have also been reported related to massive hemorrhages, stroke, and sepsis. ${ }^{11}$ Death due to cerebral infarction was reported in 1 case $(1.3 \%)$ in the study of Asano et al. ${ }^{12}$ Navani et al ${ }^{13}$ also reported a death due to sepsis after EBUS-TBNA. These reports have raised a debate on the safety of endosonography. ${ }^{14}$ The complication rates were very low in the initial studies. However, these studies included EBUS-TBNAs performed by expert bronchoscopists, and study populations were relatively small for a reliable analysis of complications. Thus, these results may be inconsistent with those of routine clinical practice. ${ }^{12,15}$ At present, the data on the true incidence of EBUS-TBNA-related complications in everyday clinical practice are scarce. The frequency of these complications is likely to increase as the technique is more widely adopted and used.

Although there are many case reports on the complications of EBUS-TBNA in the related literature, to date, there have been few investigations on the types and rates of complications related to EBUS-TBNA. The aim of the present study was to assess type and rate of serious complications related to EBUS-TBNA.

\section{Methods}

\section{Study Design}

This retrospective multi-center study was carried out between October 2008 and January 2014 in the pulmonary departments of 3 tertiary referral hospitals in Turkey. Convex-probe EBUS-TBNA was performed for staging or diagnostic purposes. A questionnaire with 15 questions was

\section{QUICK LOOK}

\section{Current knowledge}

Convex-probe endobronchial ultrasound-guided transbronchial needle aspiration (EBUS-TBNA) is a minimally invasive method for investigating the mediastinum, for staging of non-small-cell lung cancer, and for diagnosing mediastinal lesions. It is increasingly used due to safety, simplicity, high diagnostic yield, and the ability to diagnose both benign and malignant conditions.

\section{What this paper contributes to our knowledge}

Convex-probe EBUS-TBNA was safe and effective in staging and diagnosis of lung cancer in $>3,000$ procedures. Infectious complications were rare, occurring in $<0.2 \%$ of the procedures.

used and answered from subjects' medical records, including questions on age and sex of cases, the number of cases undergoing EBUS-TBNA, indications of EBUS-TBNA, anesthetic technique, total number of lymph node stations or lesions sampled by EBUS-TBNA, number of cases with non-lymph node lesions sampled by EBUS-TBNA, total number of needle passes, number of cases with serious complications, details of complications from subject background and charts, previous treatments related to underlying diseases, location of lesion or lymph node station, short axis diameter of lymph node or other lesion, final diagnosis, and treatment details of the complication (Table 1). The study protocol was approved by the local ethics committee, and written informed consent for EBUS-TBNA was obtained from all included subjects before the procedure.

\section{Serious Complications}

Lidocaine intoxication requiring special intervention, respiratory failure requiring interventions other than oxygen administration, pneumonia, mediastinitis, pericarditis, other infectious complications, fever lasting longer than $24 \mathrm{~h}$, pneumothorax requiring bed rest or thoracic drainage, prolonged bronchospasm, and hemorrhage not responsive to application of topical adrenaline and/or cold saline and requiring further intervention were considered as serious complications. However, hemorrhage other than described above; temporary laryngospasm, bronchospasm, and desaturations during the procedure; and fever lasting $<24 \mathrm{~h}$ after EBUS-TBNA were not included in the serious complications.

All of the subjects were seen at least once within $3 \mathrm{~d}$ after the EBUS procedure to receive the pathology reports, 


\section{Endobronchial Ultrasound-Guided Transbronchial NeEdle Aspiration}

Table 1. Questionnaire for Convex-Probe Endobronchial Ultrasound-Guided Transbronchial Needle Aspiration Complications

Total no. of EBUS-performed subjects

No. of EBUS-performed cases for diagnosis, staging, and diagnosis + staging

No. of EBUS-performed cases for mediastinal or parenchymal lesions Mediastinal lesions

Parenchymal lesions

No. of total lymph node stations and/or lesions where EBUS-TBNA was performed (ie, aspiration from 145 lymph node stations in 100 cases)

No. of total EBUS-TBNA aspirations (ie, 231 aspirations or passes in 100 cases)

Method of anesthesia (general or local anesthesia) and, if local anesthesia was the method, type of sedation performed

No. of EBUS-performed cases diagnosed as malignant

What were the EBUS complications?

Age and sex of the subject

What are the comorbid diseases and treatment modalities applied before EBUS (ie, radiotherapy, chemotherapy, surgery)?

Aspiration of which lymph node station was the cause of complication? Or localization of the EBUS-TBNA-performed lesion that was the cause of complication

The short axis diameter $(\mathrm{cm})$ of aspirated lymph node or lesion and number of passes

Final diagnosis of complicated case

If the complication was infection, what were the blood, aspiration, etc. culture results?

What was the medical and/or surgical treatment of complication?

Did the subject require hospitalization and/or ICU admission? For how long?

Result of the complication (death, complete recovery, partial recovery)

and they were interviewed routinely for the possibility of complications. All subjects were kept in the hospital for at least $4 \mathrm{~h}$ after EBUS-TBNA. After this period, in the presence of symptoms or complaints, a chest $\mathrm{x}$-ray was obtained. After EBUS-TBNA, a chest $\mathrm{x}$-ray was obtained in all cases with peribronchial lesions.

\section{Convex-Probe EBUS-TBNA}

Convex-probe EBUS-TBNA from hilar and mediastinal lymph nodes was performed as indicated after physical examination, routine biochemical analysis, pulmonary function tests, and thoracic computed tomography and/or positron emission tomography-integrated computed tomography were performed. EBUS-TBNA was performed in all cases as an out-patient procedure in dedicated bronchoscopy suits using convex-probe bronchoscopes (Olympus 7.5 MHz, BF-UC160F and BF-UC160F-OL8, Olympus Optical, Tokyo Japan) with an EU C2000 processor (Olympus Optical). The scope was inserted through the oral route, in supine position and under local anesthesia with lidocaine and conscious sedation with intravenous midazolam, midazolam + opioid, midazolam + propofol, or ketamine + propofol. A 22-gauge NA-201SX-4022-C needle (Olympus Optical) was used for the procedure. Short and long axis diameters of a lymph node or other lesion, station of the lymph node, and number of needle passes per subject and per lymph node or other lesion were recorded for each subject.

Convex-probe EBUS-TBNA was performed at 3 different medical centers by 6 pulmonologists. In all centers, all cases were included in the study, from the first case when EBUS-TBNA was initiated in each center to the end of the study period.

\section{Results}

Of the 3,123 cases included in the study, 861 (27.6\%) were females, and 2,262 (72.4\%) were males, with a mean age of $54.8 \pm 10.1$ (minimum 16; maximum 83) y. EBUSTBNA was performed for staging in 493 cases (15.8\%), for diagnosis in 2,109 cases (67.5\%), and for diagnosis and staging in 521 cases $(16.3 \%)$. Three hundred sixty-two $(11.6 \%)$ of the lesions sampled by EBUS-TBNA were peribronchial or intraparenchymal lesions adjacent to major airways, whereas $2,761(88.4 \%)$ were hilar or mediastinal lymphadenopathies or mediastinal lesions.

EBUS-TBNA was performed at 6,115 lymph node stations or lesions (1.96/case). There were 11,753 needle passes in 3,123 cases (3.76/case and 1.92/station or lesion). Malignancy was the final diagnosis in 1,383 cases $(44.3 \%)$, whereas it was benign disease or reactive adenitis in 1,740 cases $(55.7 \%)$.

In total, $5(0.16 \%)$ serious complications were recorded; there were no deaths. The serious complications were fever lasting longer than $24 \mathrm{~h}$, infection of bronchogenic cyst, mediastinal abscess (Figs. 1-3), pericarditis, and pneumomediastinitis with empyema. Each complication developed in a different subject. The characteristics of these subjects are given in Table 2. All subjects were treated with broad-spectrum antibiotics. Four subjects were hospitalized for $21.7 \pm 20.7 \mathrm{~d}(7-51 \mathrm{~d})$, and all subjects were discharged with improvement. We detected pneumomediastinum accompanying empyema in 1 case as a noninfectious serious complication $(0.032 \%)$.

Only 1 ( 1 of $1,383,0.072 \%)$ of these 5 cases was diagnosed with lung cancer metastasis in the lymph node sampled by EBUS-TBNA. Benign diagnoses (4 of 1,740, $0.22 \%$ ) were obtained in all other cases. A total of 10 bronchogenic cysts or cystic lesions were detected in the study population, and in 2 of these ( 2 of $10,20 \%)$ serious complications developed.

\section{Discussion}

This is the first study in Turkey regarding complications of EBUS-TBNA. To the best of our knowledge, there are 


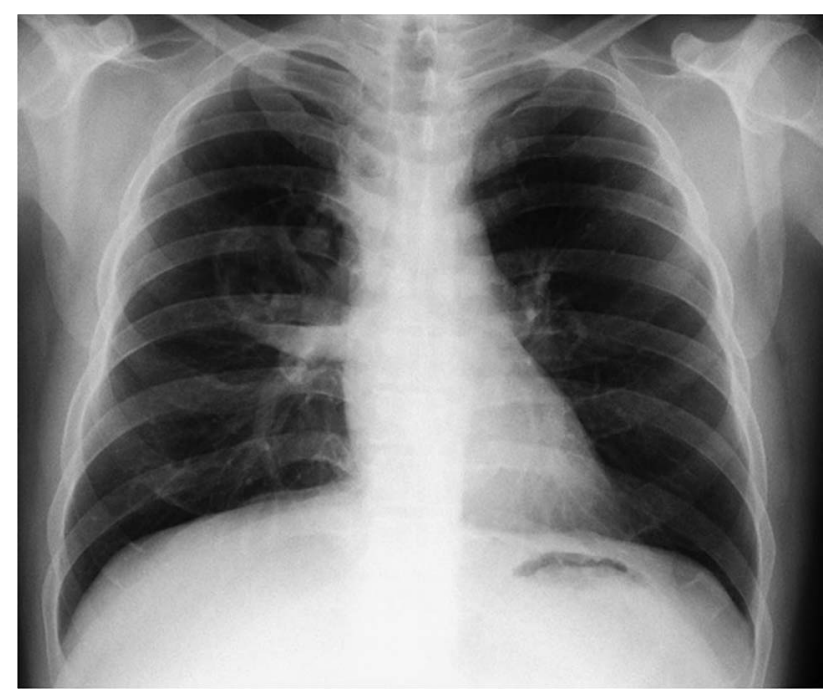

Fig. 1. Chest x-ray of the subject with mediastinal abscess.

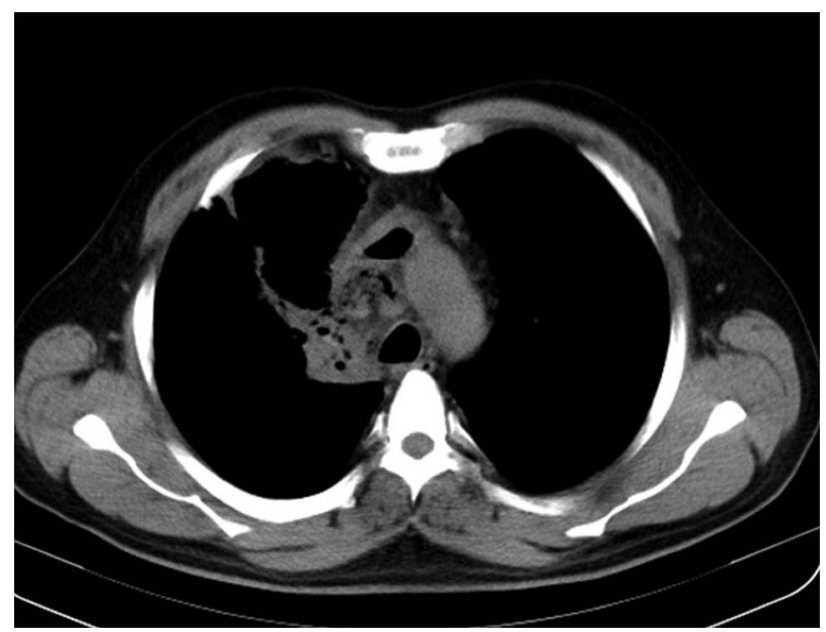

Fig. 2. Mediastinal section of thoracic computed tomogram of subject with mediastinal abscess.

2 systematic reviews and 2 Japanese survey studies in the literature describing the serious complications associated with EBUS-TBNA.2,4,12,14 The serious complication rate of EBUS-TBNA was determined to be $0.16 \%$ in the present study.

The serious complication rate of endoscopic ultrasonography and convex-probe EBUS-TBNA was reported as $0.14 \%$ in the systematic review of von Bartheld et al. ${ }^{14}$ They reported 5 serious adverse events, consisting of 2 infectious complications, 2 pneumothorax, and 1 airway edema and hypoxemia, in 9,119 EBUS-performed cases $(0.05 \%)$. There is no mortality reported either in this review or in our study. A survey study conducted in Japan revealed a complication rate of $0.46 \%$ after EBUS-TBNA of hilar and/or mediastinal lymph node lesions. ${ }^{15}$ In another Japanese survey study reported later, it was stated

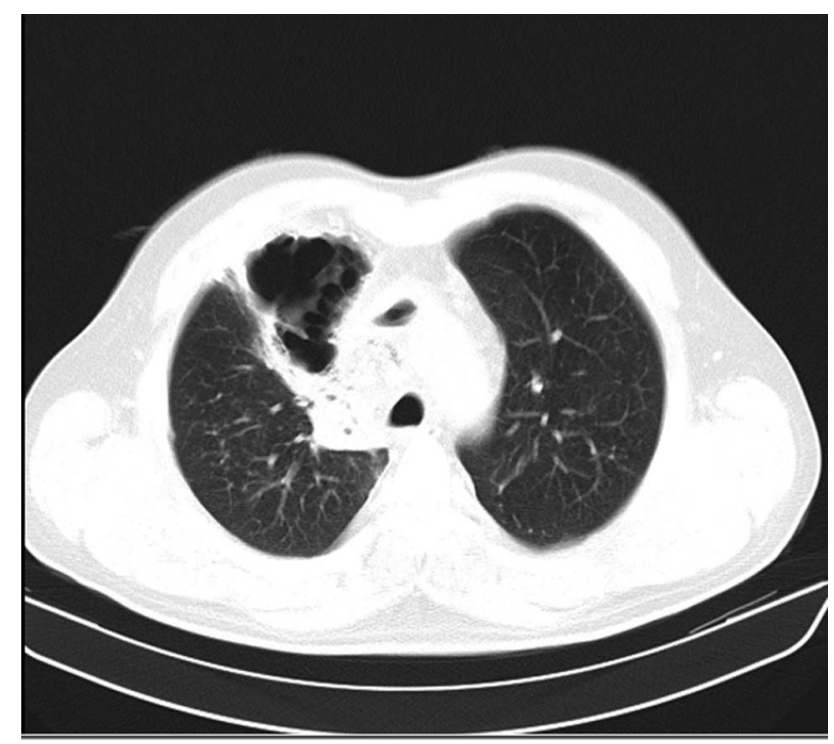

Fig. 3. Parenchymal section of thoracic computed tomogram of subject with mediastinal abscess.

that 50 detected complications $(0.68 \%)$ were hemorrhage, and $14(0.19 \%)$ were infectious complications in 7,345 cases undergoing convex-probe EBUS-TBNA. However, there was not a specific definition for serious complications in this study. It was also reported that life-threatening complications were observed in 4 cases, and a longer hospital stay was required in 14 cases. In this study, death was also reported in 1 case because of cerebral infarction (mortality rate of 0.01$).{ }^{12}$ Eapen et al ${ }^{16}$ reported a complication rate of $1.44 \%$ in their prospective study. A meta-analysis of EBUS-TBNA showed a complication rate of 2 in 1,299 cases $(0.15 \%){ }^{4}$ In this meta-analysis, only 1 case with pneumothorax requiring drainage was reported as a serious complication. However, the studies evaluated in this meta-analysis were performed by expert operators.

With the recent widespread use of EBUS-TBNA, there have been an increasing number of reports on complications associated with its use. ${ }^{5-9,12,14}$ The incidences of complications may vary depending on several factors. First, follow-up of patients to detect possible complications is not often a routine procedure, and lack of documentation is also an important factor, especially for minor complications. Second, the definition of complications and their severity can affect the rates and descriptions of the complications.

Considering the investigations and case reports reported in the literature, infectious complications constitute a significant proportion of serious complications $(5-8,12,17-19)$. In our series, all 5 serious complications were infections. Pneumomediastinum accompanying empyema was also diagnosed in 1 case.

Prolonged hospitalization was required in 4 of 5 subjects with serious complications in the current study. 


\section{Endobronchial Ultrasound-Guided Transbronchial NeEdle Aspiration}

Table 2. Features of Cases With Serious Complications

\begin{tabular}{|c|c|c|c|c|c|c|c|c|c|}
\hline Complication & Age, y & Sex & Aim & $\begin{array}{l}\text { Other } \\
\text { Diseases }\end{array}$ & Location & $\begin{array}{l}\text { LN/Lesion } \\
\text { diameter, } \\
\text { mm }\end{array}$ & Hospitalization, $\mathrm{d}$ & Culture Results & EBUS Diagnosis \\
\hline $\begin{array}{l}\text { Infection of bronchogenic } \\
\text { cyst }\end{array}$ & 44 & Female & Diagnosis & None & $\mathrm{S}$ & 42 & 7 & S. pneumonia & Bronchogenic cyst \\
\hline Fever $>24 \mathrm{~h}$ & 74 & Male & Diagnosis & $\begin{array}{l}\text { Diabetes } \\
\text { mellitus }\end{array}$ & $4 \mathrm{~L}-2 \mathrm{R}$ & $14-16$ & 7 & No growth* & Lung adenocarcinoma \\
\hline Mediastinal abscess & 49 & Male & Diagnosis & None & $4 \mathrm{R}$ & 18.5 & 22 & & Tuberculosis \\
\hline $\begin{array}{l}\text { Pneumomediastinum + } \\
\text { empyema }\end{array}$ & 78 & Male & Staging & $\begin{array}{l}\text { Prostate } \\
\text { cancer }\end{array}$ & $4 \mathrm{R}$ & 14 & 51 & S. constellatus $\dagger$ & $\begin{array}{l}\text { Anthracotic lymph } \\
\text { node }\end{array}$ \\
\hline Pericarditis & 62 & Male & Diagnosis & None & $4 \mathrm{R}$ & 22.5 & & No growth $\ddagger$ & Bronchogenic cyst \\
\hline \multicolumn{10}{|c|}{$\begin{array}{l}\text { * Pleural fluid culture. } \\
\dagger \text { Blood, urine, sputum culture. } \\
\text { † Blood culture. } \\
\text { LN = lymph node } \\
\text { EBUS = endobronchial ultrasound } \\
\mathrm{S}=\text { subcarinal } \\
\mathrm{R}=\text { right } \\
\mathrm{L}=\text { left }\end{array}$} \\
\hline
\end{tabular}

Similarly, Asano et al ${ }^{12}$ reported prolonged hospitalization in $14(15.6 \%)$ of 90 cases with complications of EBUSTBNA of a total of 7,345 cases.

It has been suggested that infectious complications occurring after EBUS-TBNA are due to seeding at the puncture site rather than bacteremia after hematogenic spread.5,12,14 In our study, Streptococcus pneumoniae and Streptococcus constellatus growths were detected on blood culture of 2 complicated cases. von Bartheld et al ${ }^{14}$ reported that cases with mediastinal cyst and sarcoidosis had more risk for infectious complications. In a study on the diagnostic methods for mediastinal benign cysts, Wildi et $\mathrm{al}^{17}$ determined that mediastinitis developed in 1 of 4 cases with mediastinal cystic lesions punctured by EBUSTBNA, whereas there were no complications reported in the remaining 3 cases receiving prophylactic antibiotics. The infection at the puncture site develops with crosscontamination of floral bacteria of the oral cavity and nasopharynx. 5,9,12 S. constellatus growing on blood culture of one of our cases is also within the floral bacteria of oral cavity and nasopharynx. In our study, EBUS-TBNA was performed on mediastinal cystic lesions in 2 (40\%) of the 5 cases with infectious complications, and there were a total of 10 cases with cystic lesions.

von Bartheld et al $^{14}$ indicated that endoscopic ultrasound and EBUS are safe techniques for an intrathoracic investigation of the mediastinum in subjects with suspected lung cancer. They reported that only 2 cases of severe infectious complications have been described in lung cancer subjects. One of them was due to technical problems, and the other was due to necrotic lymph nodes. ${ }^{14}$ In our study, the serious complication rate was $0.072 \%$ among the cases diagnosed as malignant. Previous studies have suggested that the presence of necrosis in the mediastinal lymph nodes of subjects with malignancy could be a risk factor for developing mediastinal infections due to avascularity of the lesion. ${ }^{14,20}$ We did not detect necrosis by computed tomography or EBUS in any of the lymph nodes/lesions in the complicated malignant cases of this study.

Haas $^{9}$ reported 2 infectious complications of EBUSTBNA. He pointed out that development of post-procedure infectious complications appeared to be caused by full-needle extension during EBUS-TBNA. For pericarditis occurring in one subject, he commented that full-needle extension must have allowed the needle tip to enter the pericardial space and caused pericardial contamination because the needle tip can be difficult to visualize in all planes when fully extended. 9 Our case with pericarditis had a 22.5-mm cystic paratracheal lesion. No systemic bacteremia could be shown in this case, similarly to those in the study of Haas. ${ }^{9}$

Hata et $\mathrm{al}^{21}$ reported 2 cases of tuberculosis after EBUSTBNA. One of them was complicated with the development of intrabronchial polypoid lesions at the puncture sites nearly 2 months after the EBUS-TBNA procedure at hilar and subcarinal lymph nodes. The other one had positive sputum smear for acid-fast bacilli after the EBUSTBNA, whereas she was sputum-negative before the procedure. In the present study, 1 of the 5 cases with serious complications of EBUS-TBNA developed mediastinal abscess and was also diagnosed with tuberculous lymphadenitis by EBUS-TBNA. Gupta et $\mathrm{al}^{22}$ reported a patient with tuberculous lymphadenitis in whom an endobronchial polyp developed following EBUS-TBNA. We saw neither endobronchial lesion nor sputum smear positivity after EBUS-TBNA. The subject in our study fully recovered with antibiotic treatment. Hata et $\mathrm{al}^{21}$ stated that there is a possibility of aggravation caused by puncturing the bronchial mucosa. Excessive puncturing should therefore be avoided in patients with suspected tuberculous lymphadenitis. ${ }^{21}$ Although non-infectious serious complications, such as needle breakage, hemorrhage, intramural hema- 


\section{Endobronchial Ultrasound-Guided Transbronchial NeEdle Aspiration}

toma of the pulmonary artery, and hemopneumomediastinum, have also been reported in the related literature, we only encountered infectious complications.

The limitations of this study are its retrospective design and lack of long-term follow-up of all cases for the assessment of complications after EBUS-TBNA. It is possible that both of these limitations could lead to underestimation of the complication rate yet, but it is unlikely that the overall rate would be significantly different.

\section{Conclusions}

In conclusion, convex-probe EBUS-TBNA is a safe method in general but infrequently can cause serious infections. All precautions should be taken for complications before and during the procedure. After evaluation of the results of our study and the data obtained from the literature, we recommend to avoid performing EBUS-TBNA on mediastinal cystic lesions or to use a prophylactic antibiotic if it is necessary to perform EBUS-TBNA.

\section{REFERENCES}

1. Detterbeck FC, Jantz MA, Wallace M, Vansteenkiste J, Silvestri GA. Invasive mediastinal staging of lung cancer: ACCP evidence-based clinical practice guidelines (2nd edition). Chest 2007;132(3 Suppl): 202S-220S

2. Varela-Lema L, Fernández-Villar A, Ruano-Ravina A. Effectiveness and safety of endobronchial ultrasoundtransbronchial needle aspiration: a systematic review. Eur Respir J 2009;33(5):1156-1164.

3. Özgül MA, Çetinkaya E, Tutar N, Özgül G. An unusual complication of endobronchial ultrasound-guided transbronchial needle aspiration (EBUS-TBNA): the needle breakage. Ann Thorac Cardiovasc Surg 2014;20(Suppl):567-569.

4. Gu P, Zhao YZ, Jiang LY, Zhang W, Xin Y, Han BH. Endobronchial ultrasound-guided transbronchial needle aspiration for staging of lung cancer: a systematic review and meta analysis. Eur J Cancer 2009; 45(8):1389-1396.

5. Huang CT, Chen CY, Ho CC, Yu CJ. A rare constellation of empyema, lung abscess, and mediastinal abscess as a complication of endobronchial ultrasound-guided transbronchial needle aspiration. Eur J Cardiothorac Surg 2011;40(1):264-265.

6. Moffatt-Bruce SD, Ross P Jr. Mediastinal abscess after endobronchial ultrasound with transbronchial needle aspiration: a case report. J Cardiothorac Surg 2010;5:33.

7. Parker KL, Bizekis CS, Zervos MD. Severe mediastinal infection with abscess formation after endobronchial ultrasound-guided transbrochial needle aspiration. Ann Thorac Surg 2010;89(4):1271-1272.

8. Botana-Rial M, Núñez-Delgado M, Pallarés-Sanmartin A, LeiroFernández V, Represas Represas C, González Silvia AI, FernándezVillar A. Intramural hematoma of the pulmonary artery and hemo- pneumomediastinum after endobronchial ultrasound-quided transbronchial needle aspiration. Respiration 2012;83(4):353-356.

9. Haas AR. Infectious complications from full extension endobronchial needle aspiration. Eur Respir J 2009;33(4):935-938.

10. Yasufuku K. Complications of endobronchial ultrasound-guided transbronchial needle aspiration. J Bronchology Interv Pulmonol 2010;17(4):287-288.

11. Al-Qadi MO, Maldonado F. Focal tracheal stenosis due to intramural hematoma following endobronchial ultrasound-guided transbronchial needle aspiration. J Bronchology Interv Pulmonol 2014;21(3):274276.

12. Asano F, Aoe M, Ohsaki Y, Okada Y, Sasada S, Sato S, et al Complications associated with endobronchial ultrasound-guided transbronchial needle aspiration: a nationwide survey by the Japan Society for Respiratory Endoscopy. Respir Res 2013;14:50.

13. Navani N, Brown JM, Nankivell M, Woolhouse I, Harrison RN, Jeebun V, et al. Suitability of endobronchial ultrasound-guided transbronchial needle aspiration specimens for subtyping and genotyping of non-small cell lung cancer: a multicenter study of 774 patients Am J Respir Crit Care Med 2012;185(12):1316-1322.

14. von Bartheld MB, van Breda A, Annema JT. Complication rate of endosonography (endobronchial and endoscopic ultrasound): a systematic review. Respiration 2014;87(4):343-351.

15. Asano F, Aoe M, Ohsaki Y, Okada Y, Sasada S, Sato S, et al. Deaths and complications associated with respiratory endoscopy: a survey by the Japan Society for Respiratory Endoscopy in 2010. Respirology 2012;17(3):478-485.

16. Eapen GA, Shah AM, Lei X, Jimenez CA, Morice RC, Yarmus L, et al. Complications, consequences and practice patterns of endobronchial ultrasound-guided transbronchial needle aspiration results of the AQuIRE registry. Chest 2013;143(4):1044-1053.

17. Wildi SM, Hoda RS, Fickling W, Schmulewitz N, Varadarajulu S, Roberts SS, et al. Diagnosis of benign cysts of the mediastinum: the role and risks of EUS and FNA. Gastrointest Endosc 2003;58(3): 362-368.

18. Gochi F, Chen F, Aoyama A, Date H. Mediastinal infectious complication after endobronchial ultrasound-guided transbronchial needle aspiration. Interact Cardiovasc Thorac Surg 2013;17(4):751-752.

19. Oguri T, Imai N, Imaizumi K, Elshazley M, Hashimoto I, Hashimoto $\mathrm{N}$, Hasegawa Y. Febrile complications afterendobronchial ultrasoundguided transbronchial needle aspiration for intrapulmonary mass lesions of lung cancer-a series of 3 cases. Respir Investig 2012;50(4): 162-165.

20. Aerts JG, Kloover J, Los J, van der Heijden O, Janssens A, Tournoy KG. EUS-FNA of enlarged necrotic lymph nodes may cause infectious mediastinitis. J Thorac Oncol 2008;3(10):1191-1193.

21. Hata Y, Sakamoto S, Otsuka H, Sato K, Sato F, Makino T. EBUSTBNA related complications in a patient with tuberculous lymphadenopathy. Intern Med 2013;52(22):2553-2559.

22. Gupta R, Park HY, Kim H, Um SW. Endobronchial inflammatory polyp as a rare complication of endobronchial ultrasoundtransbronchial needle aspiration. Interact Cardiovasc Thorac Surg 2010;11(3): 340-341. 